\title{
Genome-wide study of ER-regulated IncRNAs shows AP000439.3 may function as a key regulator of cell cycle in breast cancer
}

\author{
YIN ZHANG $^{1 *}$, DAN-LAN WANG ${ }^{1 *}$, HAI-YAN YAN $^{1 *}$, JIAN-YOU LIAO $^{1}$, \\ JIE-HUA HE ${ }^{1}$, KAI-SHUN HU ${ }^{1}$, WEI-XI DENG ${ }^{1}$, YAN-JIE WANG ${ }^{1}$, \\ HONG-TAO XING ${ }^{2}$, H. PHILLIP KOEFFLER ${ }^{2-4}$ and DONG YIN ${ }^{1}$ \\ ${ }^{1}$ Guangdong Provincial Key Laboratory of Malignant Tumor Epigenetics and Gene Regulation, \\ Research Center of Medicine, Sun Yat-Sen Memorial Hospital, Sun Yat-Sen University, \\ Guangzhou, Guangdong 510120, P.R. China; ${ }^{2}$ Cedars-Sinai Medical Center, \\ Division of Hematology/Oncology, University of California Los Angeles School of Medicine, \\ Los Angeles, CA, USA; ${ }^{3}$ Cancer Science Institute of Singapore, National University of Singapore; \\ ${ }^{4}$ National University Cancer Institute, National University Hospital, Singapore, Republic of Singapore
}

Received February 18, 2017; Accepted July 12, 2017

DOI: $10.3892 / o r .2017 .5975$

\begin{abstract}
Estrogen receptor (ER) plays important roles in cell growth, development and tumorigenesis. Although ER-regulated genes have been extensively investigated, little is known about roles of ER-regulated IncRNAs in breast cancer. Here, we conducted genome-wide study of ER-regulated lncRNAs by using RNA-seq, ChIP-seq and TCGA data. A total of identified 114 ER-regulated lncRNAs were identified, many of them were overexpressed in $\mathrm{ER}^{+}$breast cancer and co-expressed with some key regulators. Silencing one of most prominent lncRNA, AP000439.3, resulted in inhibition of cell cycle progression and proliferation. Further study revealed AP000439.3 can regulate expression of CCND1 through enhancing estrogen receptor induction of CCND1. This finding revealed lncRNAs may serve
\end{abstract}

Correspondence to: Professor Dong Yin, Guangdong Provincial Key Laboratory of Malignant Tumor Epigenetics and Gene Regulation, Research Center of Medicine, Sun Yat-Sen Memorial Hospital, Sun Yat-Sen University, Guangzhou, Guangdong 510120, P.R. China

E-mail: yin_dong@yahoo.com

${ }^{*}$ Contributed equally

Abbreviations: ER, estrogen receptor; lncRNA, long non-coding RNA; EREs, estrogen response elements; lincRNA, long intergenic non-coding RNA; DE, differential expression; qPCR, quantitative polymerase chain reaction; TCGA, The Cancer Genome Atlas; RPM, reads per million; FPKM, reads per kilobase of exon model per million mapped reads; ChIP, chromatin immunoprecipitation

Key words: estrogen receptor, lncRNA, breast cancer, CCND1, AP000439.3 as important effectors of ER in regulation of gene expression and cell phenotype in breast cancer.

\section{Introduction}

Estrogen plays critical roles in normal mammary gland development, physiological processes as well as tumorigenesis. Estrogen receptor (ER) is the major mediator of the effect of estrogen. After binding to estrogen, ER is activated and translocates into the cell nucleus (1). In the nucleus, ER can transcriptionally regulate target genes either by directly binding to estrogen response elements (EREs) or through protein-protein interactions with other transcriptional factors such as AP1, SP1 and NF- $\kappa$ B (2). Moreover, 70\% of breast cancers express ER. ER is essential for the initiation and development of breast cancers $(1,3,4)$. The pro-oncogenic effect of ER is mediated primarily by transcriptionally regulating its target genes. Various genes that play crucial roles in cell proliferation and apoptosis such as $c-M y c, C C N D 1$ and $B C L 2$ have been identified as ER-regulated genes. Hundreds of proteincoding genes have been identified as ER-regulated genes by using microarray and RNA-seq as well as ChIP-seq (5-9).

Long non-coding RNAs (lncRNAs) refer to non-coding RNAs consisting of $>200$ nucleotides. Recently, the important roles of lncRNAs in breast cancer cell proliferation, apoptosis, metastasis and endocrine resistance have been reported (10-12). Because of their crucial roles in breast cancer, we explored ER-regulated lncRNAs. Although a few studies have been carried out to identify ER-regulated lncRNAs very recently (13-15), their roles in regulation of gene expression and cell phenotype remains largely unknown. Here, we report a genome-wide study of ER-regulated lncRNAs by conducting an integrate analysis of ChIP-seq, strand-specific RNA-seq and TCGA clinical data. We observed that many of these ER-regulated lncRNAs were overexpressed in $\mathrm{ER}^{+}$ 
breast cancer and exhibited co-expression with several key regulator proteins. Moreover, we found one of most prominent lncRNAs, AP000439.3, can promote cell cycle progression through enhancing $C C D N 1$ expression induced by estrogen. These findings reveal ER can regulate many lncRNAs that exhibit important functions in regulation of gene expression and cell phenotype in breast cancer.

\section{Materials and methods}

Datasets and computational analysis. The 17 $\beta$-estradiol (E2) and vehicle treated MCF7 RNA-seq data (pair-end, strand-specific) was previously reported by Dago et al (16) and obtained from GEO dataset (GSE64590). For RNA-seq analysis, the sequenced reads were aligned to human reference genome (Hg38) and transcriptome (GENCODE.v23) using STAR (17) and then processed by RSEM (18). Genes with FDR $<0.05$ generated by both DESeq (19) and edgeR (20) were considered as differentially expressed genes.

ER $\alpha$ ChIP-seq of estrogen treated and untreated MCF7 were reported by Franco et al (21) and obtained from GEO dataset (GSE59530). Reads of ChIP-seq were aligned to human reference genome $(\mathrm{Hg} 38)$ using BOWTIE (22). The estrogen treated peaks were generated by MACS (23) using untreated as control. The ER binding sites within $\pm 100 \mathrm{~kb}$ region of transcriptional start sites (TSS) of protein-coding genes and IncRNAs (GENCODE.v23) were considered as potential ER regulatory sites.

The TCGA lncRNA data were downloaded from TANRIC database (24), mRNA and RPPAs data were downloaded from Broad Dashboard-Stddata (https://confluence.broadinstitute. org/display/GDAC/Dashboard-Stddata).

Cell culture and treatments. MCF7, ZR-75-1 and T47D cells were obtained from ATCC. MCF7 cells were cultured in Eagle's minimum essential medium supplemented with $10 \% \mathrm{FBS}$; ZR-75-1 and T47D were cultured in RPMI-1640 media supplemented with $10 \%$ FBS. Before estrogen treatments, the cells were grown for $72 \mathrm{~h}$ in phenol red-free MEM Eagle medium supplemented with $10 \%$ charcoal-dextran-treated FBS. The cells were then treated with ethanol (vehicle) or $10 \mathrm{nM}$ E2 for 24 h. siRNAs were synthesized in GenePharma Co. (Shanghai, China). The sequences of siRNA are listed in Table II. siRNA was transfected at a final concentration of $50 \mathrm{nM}$ using Lipofectamine 2000 reagent (Invitrogen).

Real-time quantitative PCR and western blotting. Cells were grown and treated as described above and then RNA was collected using TRIzol reagent (Invitrogen), RNA was reversetranscribed using PrimeScript RT reagent kit (Takara RR047A) and analyzed by LightCycler ${ }^{\circledR} 96$ real-time PCR thermocycler (Roche), primers are listed in Table II. The lncRNA expression was normalized to $\beta$-actin transcript as an internal standard. The CCND1 and $\alpha$-tubulin protein were detected by western blotting using cyclin D1 mouse mAb (BD Pharmigen \#556470) and $\alpha$-tubulin antibody (Santa Cruz, SC-5286).

Cell cycle analysis and colony formation assay. Seventy-two hours after siRNA transfection, cells were digested and washed with PBS and then fixed in $70 \%$ ethanol overnight at $4^{\circ} \mathrm{C}$, then incubated with RNase and propidium iodide (PI) for $10 \mathrm{~min}$ and analyzed by flow cytometry.

For colony forming analysis, 1,000 cells were plated in 6-well plates and grown for 2 weeks, colonies were fixed with $4 \%$ paraformaldehyde and stained with $0.1 \%$ crystal violet. The number of colonies were counted and analyzed by ImageJ software.

Chromatin immunoprecipitation (ChIP) assay. The ChIP assay were performed as previously described (25) with MCF7 cells using $3 \mu \mathrm{g}$ normal IgG (CST, \#2729) and ER (Santa Cruz sc-543) antibody. The ChIP PCR primers were listed in Table II.

Statistical analysis. Wilcoxon rank-sum test was used for comparing fold change between lncRNA and protein. Spearman's rank moment correlation coefficient was calculated for analysis of the co-expression of lncRNAs and RPPAs, Pearson's product moment correlation for lncRNA-mRNA co-expression. For qRT-PCR and colony formation assay, Student's t-test was used to test for statistical significance of the differences between the different group parameters. p-values of $<0.05$ was considered statistically significant.

\section{Results}

Global identification and characterization of ER-regulated lncRNAs. To identify ER-regulated IncRNAs in a genome-wide manner, we exploited published RNA-seq of E2- and vehicle-treated cells that were originally used for analyzing alternative mRNA splicing (16) (GSE64590). We chose this dataset because: i) this RNA-seq was strand-specific, which was superb for analyzing antisense transcripts that overlapped with host genes. ii) Three repetitions with high reproducibility occurred in this dataset. We conducted an analysis of differential expression (DE) transcripts using edgeR (20) and DEseq (19) processed by RSEM (18). We observed 2,869 DE transcripts, which included 1,784 upregulated and 1,085 downregulated transcripts. The dominant types of DE transcripts were protein-coding genes (92.5\%); 147 lncRNAs were found to express differentially upon E2 treatment (Fig. 1A). Of these lncRNAs, $45.6 \%$ were long intergenic RNA (lincRNAs), $19.7 \%$ were antisense and $16.3 \%$ were processed transcripts (Fig. 1B). DE IncRNAs showed significantly lower expression than protein-coding genes (Fig. 1C), which is in agreement with previous reports (26). Despite low expression of DE lncRNAs, we found DE lncRNAs exhibited a higher fold-change than DE protein-coding genes in the presence of estrogen (Fig. 1D). LncRNAs tend to be more specifically expressed than protein $(27,28)$; moreover, lncRNAs differ not just between tissues, but also between closely related cell types, which indicates lncRNAs are likely under stricter regulations (29). In agreement with this notion, our analysis revealed lncRNAs were under more rigorous regulation of activated ER. To investigate whether these DE transcripts were transcriptionally regulated by ER, we conducted an analysis of ER binding sites using published ChIP-seq data, and 114 of 147 (77.6\%) DE lncRNAs had at least one ER binding site within their genomic domain (within $\pm 100 \mathrm{~kb}$ from TSS). Half of these lncRNAs were upregulated by E2 and these lncRNAs were 
Table I. List of ER-upregluated lncRNAs.

\begin{tabular}{|c|c|c|c|}
\hline Ensembl ID & LncRNA name & Fold change $(\log 2)$ & FDR \\
\hline ENSG00000273565.1 & CTD-3075F15.1 & 5.13 & 0.000258667 \\
\hline ENSG00000249346.6 & LINC01016 & 4.30 & $1.89 \mathrm{E}-107$ \\
\hline ENSG00000266036.1 & RP11-452I5.2 & 4.04 & $3.29 \mathrm{E}-06$ \\
\hline ENSG00000254290.1 & RP11-150O12.3 & 2.74 & $6.46 \mathrm{E}-08$ \\
\hline ENSG00000258354.1 & MIR3180-1 & 2.69 & $6.93 \mathrm{E}-06$ \\
\hline ENSG00000272472.1 & RP11-95G17.2 & 2.51 & $5.95 \mathrm{E}-15$ \\
\hline ENSG00000244265.1 & SIAH2-AS1 & 2.48 & 0.000112224 \\
\hline ENSG00000227036.6 & LINC00511 & 1.97 & $5.34 \mathrm{E}-30$ \\
\hline ENSG00000259459.5 & RP11-321G12.1 & 1.86 & 0.000182177 \\
\hline ENSG00000229525.1 & AC053503.4 & 1.83 & $3.17 \mathrm{E}-06$ \\
\hline ENSG00000233885.7 & YEATS2-AS1 & 1.48 & $2.03 \mathrm{E}-14$ \\
\hline ENSG00000259080.1 & RP11-158I13.2 & 1.46 & $1.04 \mathrm{E}-05$ \\
\hline ENSG00000261578.1 & RP11-21L23.2 & 1.39 & $1.73 \mathrm{E}-26$ \\
\hline ENSG00000280186.1 & RP11-483I13.6 & 1.35 & $9.59 \mathrm{E}-53$ \\
\hline ENSG00000204792.2 & LINC01291 & 1.32 & $1.38 \mathrm{E}-21$ \\
\hline ENSG00000253125.1 & RP11-459E5.1 & 1.24 & $6.02 \mathrm{E}-05$ \\
\hline ENSG00000260401.1 & RP11-800A3.4 & 1.23 & $2.02 \mathrm{E}-94$ \\
\hline ENSG00000261051.1 & RP11-274H2.5 & 1.22 & $1.07 \mathrm{E}-16$ \\
\hline ENSG00000266709.1 & RP11-21401.2 & 1.19 & $1.26 \mathrm{E}-05$ \\
\hline ENSG00000223749.7 & MIR503HG & 1.18 & $2.99 \mathrm{E}-42$ \\
\hline ENSG00000280924.1 & LINC00628 & 1.16 & $2.97 \mathrm{E}-05$ \\
\hline ENSG00000226471.6 & CTA-292E10.6 & 1.16 & $9.45 \mathrm{E}-19$ \\
\hline ENSG00000277159.1 & RP11-88E10.4 & 1.12 & $2.90 \mathrm{E}-09$ \\
\hline ENSG00000281207.1 & SLFNL1-AS1 & 1.03 & $6.93 \mathrm{E}-06$ \\
\hline ENSG00000280073.1 & CTD-2525P14.5 & 0.95 & $1.47 \mathrm{E}-05$ \\
\hline ENSG00000232956.8 & SNHG15 & 0.93 & $1.38 \mathrm{E}-37$ \\
\hline ENSG00000230836.1 & LINC01293 & 0.84 & $5.77 \mathrm{E}-15$ \\
\hline ENSG00000246174.7 & KCTD21-AS1 & 0.79 & $3.09 \mathrm{E}-08$ \\
\hline ENSG00000236144.6 & TMEM147-AS1 & 0.42 & $8.75 \mathrm{E}-07$ \\
\hline ENSG00000213062.4 & RP1-206D15.6 & 0.75 & $1.69 \mathrm{E}-05$ \\
\hline ENSG00000270344.2 & RP11-734K2.4 & 0.73 & $9.31 \mathrm{E}-17$ \\
\hline ENSG00000260257.2 & RP5-1085F17.3 & 0.72 & 0.000169607 \\
\hline ENSG00000261226.1 & RP11-830F9.7 & 0.63 & 7.69E-06 \\
\hline ENSG00000249859.7 & PVT1 & 0.62 & $5.88 \mathrm{E}-46$ \\
\hline ENSG00000271020.1 & RP11-10C24.1 & 0.61 & $6.26 \mathrm{E}-05$ \\
\hline ENSG00000247092.6 & SNHG10 & 0.57 & $2.45 \mathrm{E}-12$ \\
\hline ENSG00000232445.1 & RP11-132A1.4 & 0.57 & $2.35 \mathrm{E}-06$ \\
\hline ENSG00000271643.1 & RP11-10C24.3 & 0.56 & $9.12 \mathrm{E}-08$ \\
\hline ENSG00000262202.4 & RP11-160E2.6 & 0.56 & 5.30E-09 \\
\hline ENSG00000259977.1 & AL121578.2 & 0.53 & 7.72E-06 \\
\hline ENSG00000255774.1 & AP000439.3 & 0.40 & 0.000181605 \\
\hline ENSG00000252690.3 & SCARNA15 & 0.39 & $1.54 \mathrm{E}-08$ \\
\hline ENSG00000255717.6 & SNHG1 & 0.34 & $6.40 \mathrm{E}-61$ \\
\hline ENSG00000236824.1 & BCYRN1 & 0.32 & 4.03E-08 \\
\hline ENSG00000236830.6 & CBR3-AS1 & 0.31 & $6.86 \mathrm{E}-12$ \\
\hline ENSG00000226950.6 & DANCR & 0.30 & $7.63 \mathrm{E}-15$ \\
\hline ENSG00000234912.9 & SNHG20 & 0.25 & $3.78 \mathrm{E}-05$ \\
\hline ENSG00000227051.5 & C14orf132 & 0.23 & $3.24 \mathrm{E}-16$ \\
\hline
\end{tabular}


Table I. Continued.

\begin{tabular}{llcc}
\hline Ensembl ID & LncRNA name & Fold change (log2) & FDR \\
\hline ENSG00000235123.5 & DSCAM-AS1 & 0.22 & $2.09 \mathrm{E}-29$ \\
ENSG00000163597.14 & SNHG16 & 0.20 & $3.35 \mathrm{E}-46$ \\
ENSG00000259623.1 & RP11-156E6.1 & 0.19 & $1.91 \mathrm{E}-06$ \\
ENSG00000260032.1 & LINC00657 & 0.16 & $3.44 \mathrm{E}-37$ \\
ENSG00000224032.6 & EPB41L4A-AS1 & 0.16 & $5.66 \mathrm{E}-06$ \\
ENSG00000242125.3 & SNHG3 & 0.14 & $1.29 \mathrm{E}-05$ \\
ENSG00000244879.4 & GABPB1-AS1 & 0.11 & $1.09 \mathrm{E}-05$ \\
ENSG00000234741.7 & GAS5 & 0.06 & $6.73 \mathrm{E}-08$ \\
ENSG00000175061.17 & LRRC75A-AS1 & 0.05 & $3.01 \mathrm{E}-05$ \\
\hline
\end{tabular}

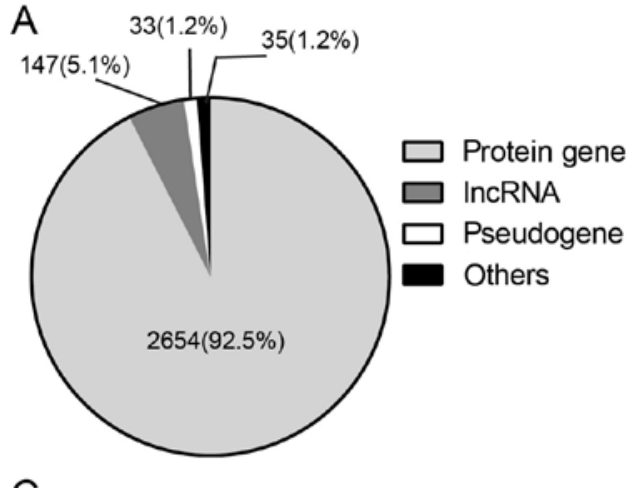

C

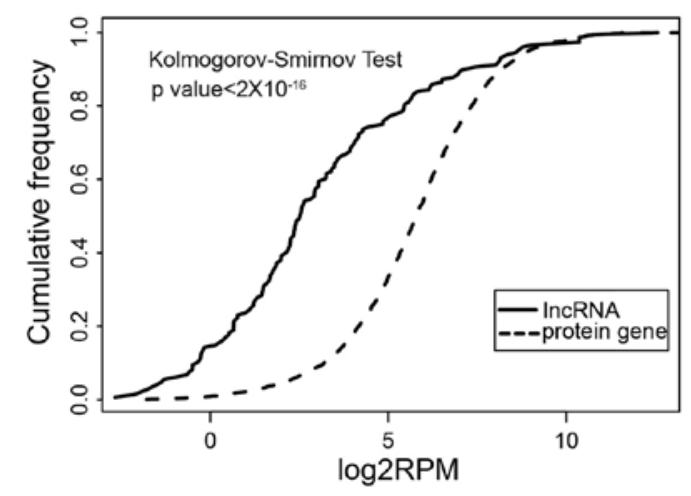

B

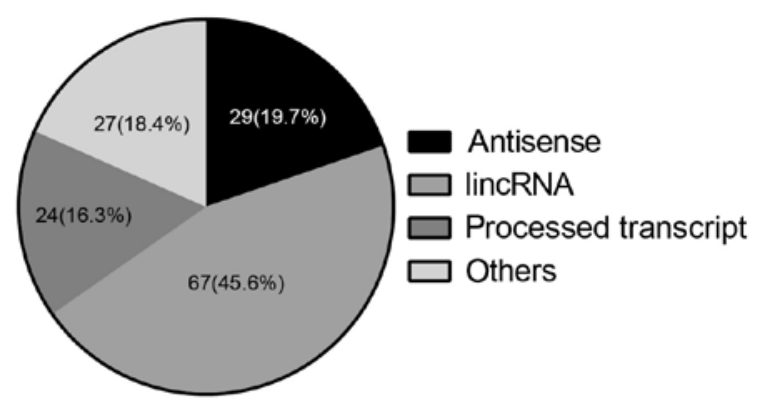

D

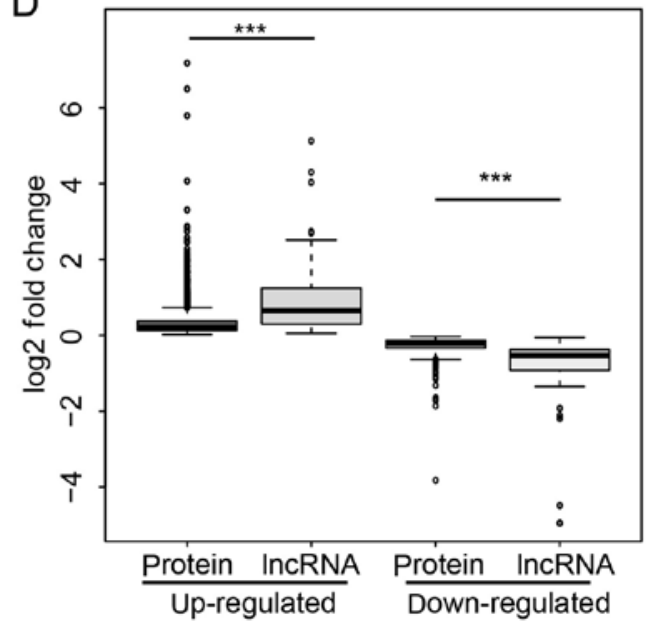

Figure 1. Summary of differential expressed lncRNAs upon E2 treatment. (A) Distribution of differential expressed transcriptions upon estrogen treatment. 'Others' include snoRNAs, snRNAs and miscRNAs. (B) Fraction of different classification of DE lncRNAs. (C) Cumulative frequency distribution showing expression levels of DE lncRNAs and protein-coding genes. RPM (reads per million) value are on log scale. (D) Boxplots showing fold change of DE lncRNAs compared to protein-coding genes. Significance calculated by Wilcoxon Rank-Sum test indicated by ${ }^{*} \mathrm{p}<0.05,{ }^{* *} \mathrm{p}<0.01$ and ${ }^{* * * *} \mathrm{p}<0.001$.

referred to as putative ER-upregulated lncRNAs (Table I). To explore the potential roles of these IncRNAs, we conducted co-expression analysis between IncRNAs and reverse-phase protein array (RPPAs) proteomics data (30), which includes extensively validated antibodies to nearly 200 proteins and phosphoproteins of TCGA clinical samples. We found most of these lncRNAs co-expressed with some key regulators of the cell cycle (cyclin family) and apoptosis (Bcl-2 family) as well as key components of important signaling pathway such as IGF1R, MAPK (pT202/Y204) and JNK2. These data suggest that ER can transcriptionally regulate hundreds of lncRNAs that may participate in some important cellular processes.

Detection of ER-regulated lncRNAs. To verify the DE IncRNAs analyzed by RNA-seq, we selected six lncRNAs for further analysis. We first performed qPCR experiments after E2 treatment. Consistent with RNA-seq results. In MCF7, the most significantly changed lncRNA is AP000439.3, which increased 6 times. The expressions of this lncRNA in T47D was too low to be effectively detected. We also observed 
Table II. Primers and siRNAs used in this study.

\begin{tabular}{ll} 
qPCR primers & \multicolumn{1}{c}{ Forward } \\
\hline AP000439.3 & CCCCAGGCTAGGAAGATGT \\
RP11-321G12.1 & GGTTTGGTTCCCAATTGTTG \\
RP11-150012.3 & ACCATTTCCAAACTGCCAAG \\
LINC01016 & TACAGCATGGTTCCCAAATG \\
SIAH2-AS1 & CTCCTCAATCCCCACACAGT \\
RP11-95G17.2 & TTGCTGTAGTGCGGCTTAAA
\end{tabular}

ChIP PCR primers
Forward

AGGGAGAGTTCCCAGGAGTC CTCCACCGAGCACTCCATAC
Reverse

GAGCCAAGAGGTCCTCACAG TTGGAAGACCCCATCTTCAC GCTCCATGCACACTCAAGAA GGGCCATGGTCACTCATATT TGCAGACGTGTATTCGGGTA TAACCCCTTGCAATCAGCTC

ER binding site 1

ER binding site 2

sense

antisene

siRNAs

GCUAGGAAGAUGUGCACCU
GCAAAGCUCACAGGAAAUA
CGAGUAUGAUCCUACCAGA

AGGUGCACAUCUUCCUAGC

AP000439.3 siRNA 1\#

AP000439.3 siRNA 2\#

CGAGUAUGAUCCUACCAGA

ER $\alpha$ siRNA

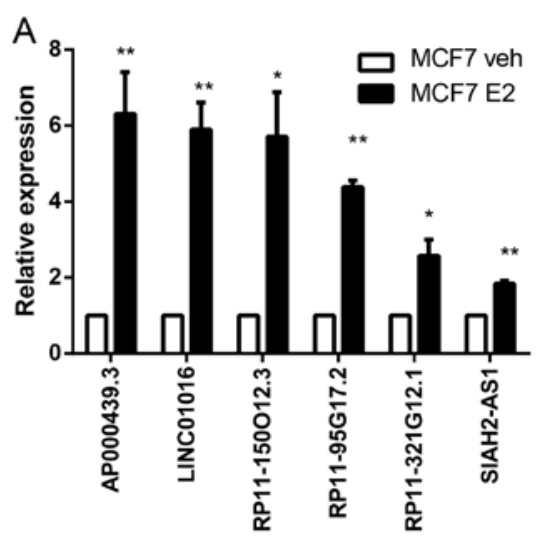

B

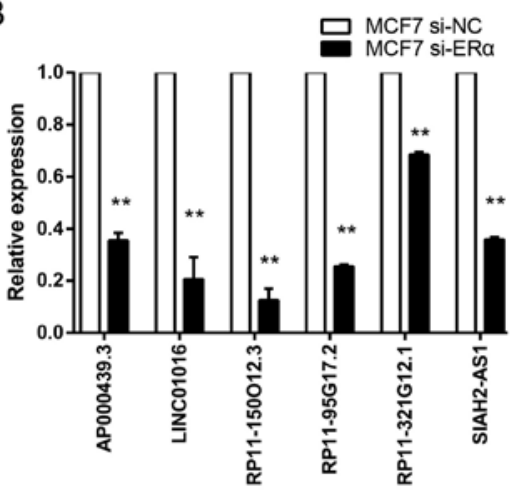

C

D

CAGCCCTGTCTGAGCAATTT TGCCTCTTGTTTCCCCTAAA

Reverse

UAUUUCCUGUGAGCUUUGC

UCUGGUAGGAUCAUACUCG
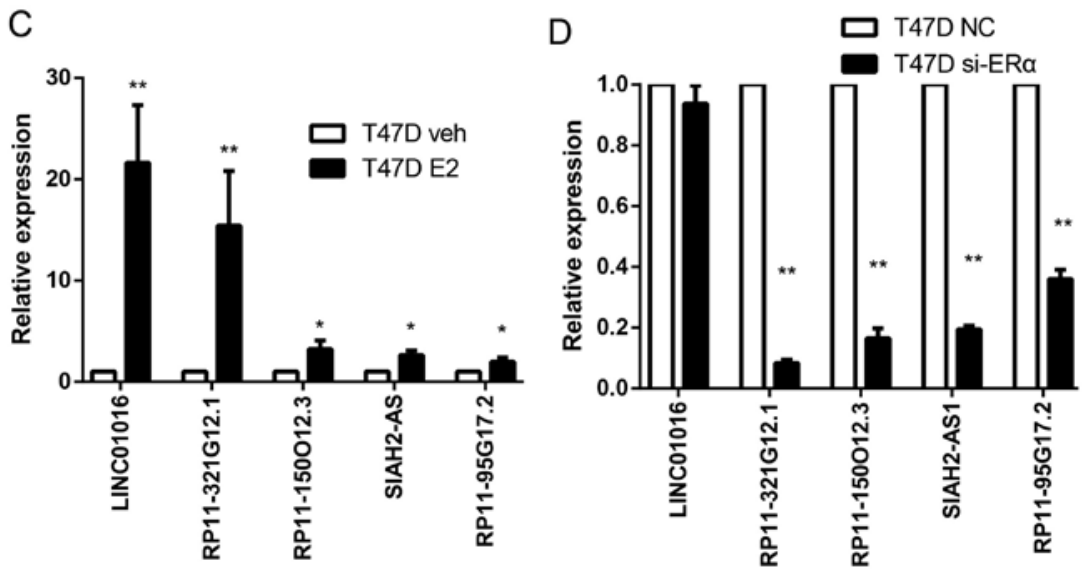

Figure 2. Expression change of ER target lncRNAs followed by either E2 treatment or knock down of ER $\alpha$. (A) MCF7 cells were treated with $10 \mathrm{nM}$ E2 or vehicle (ethanol) for $24 \mathrm{~h}$ and expression of lncRNAs were detected by qPCR. (B) MCF7 cells were transfected with either ER $\alpha$ or control siRNAs for $72 \mathrm{~h}$ and IncRNAs were detected by qPCR. (C) T47D cells were treated with $10 \mathrm{nM} \mathrm{E2}$ or vehicle (ethanol) for $24 \mathrm{~h}$ and expression of lncRNAs was detected by qPCR. (D) T47D cells were transfected with ER $\alpha$ siRNAs for $72 \mathrm{~h}$ and lncRNAs were detected by qPCR. Significance calculated by Student's t-test is indicated by ${ }^{*} \mathrm{p}<0.05,{ }^{* *} \mathrm{p}<0.01$ and ${ }^{* * *} \mathrm{p}<0.001$.

significant increase of $A P 000439.3$ after E2 treatment in ZR-75-1 cells (data not shown). The other lncRNAs also increased remarkably after E2 treatment of both in MCF7 and T47D (Fig. 2A and C). To confirm that these DE lncRNAs 

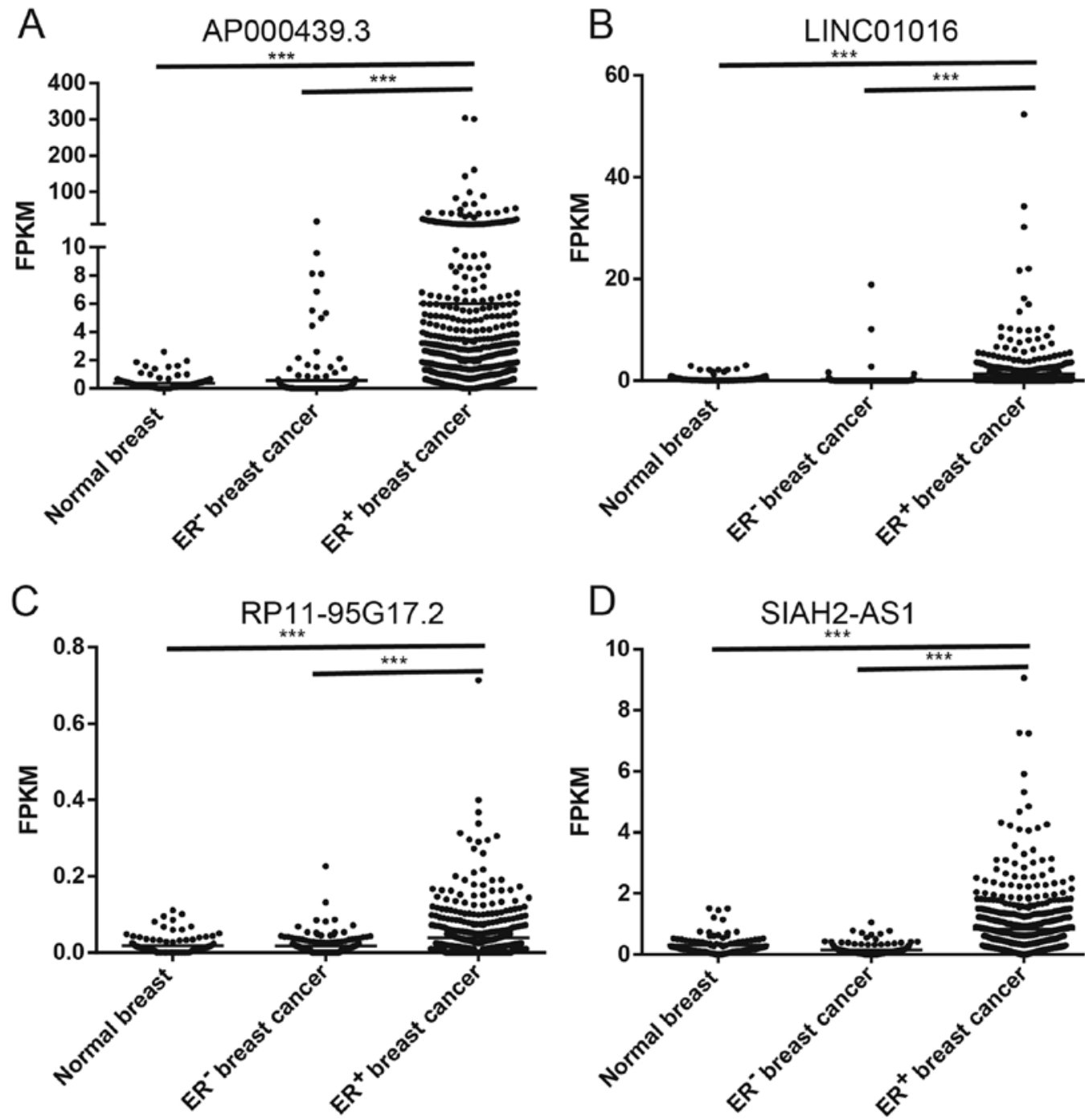

$E$

RP11-150012.3
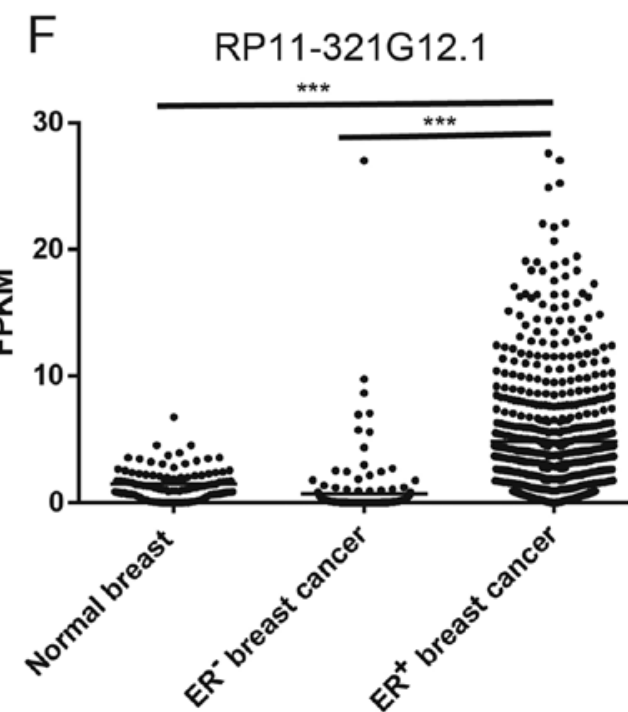

Figure 3. Expression of selected lncRNAs in clinical samples. The expression (FPKM) of these lncRNAs in breast cancer were shown by using TCGA data Significance calculated by Wilcoxon rank-sum test indicated by ${ }^{*} \mathrm{p}<0.05,{ }^{* *} \mathrm{p}<0.01$ and ${ }^{* * * *} \mathrm{p}<0.001$.

are regulated by ER, we silenced ER $\alpha$ in MCF7 and T47D by siRNA. In MCF7 cells, all of these lncRNAs dramatically decreased when silencing ER $\alpha$, some of the lncRNAs such as RP11-150O12.3, AP000439.3 and RP11-95G17.2 decreased by $>60 \%$ (Fig. 2B and D). Similar results were observed when ER was knocked down in T47D. Although LINC01016 is a significantly upregulated lncRNA in T47D, silencing ER did not result in its reduction. LINC01016 has been reported as 

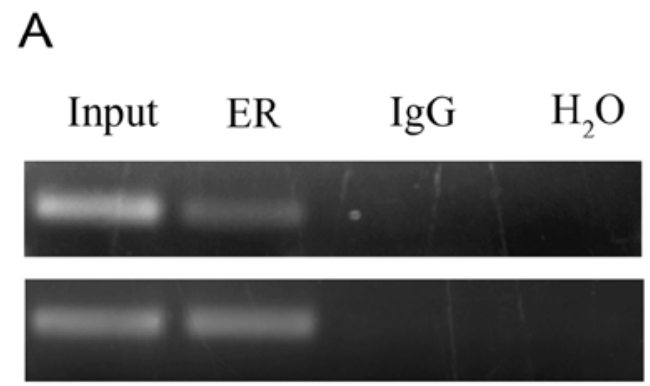

ER binding site 2
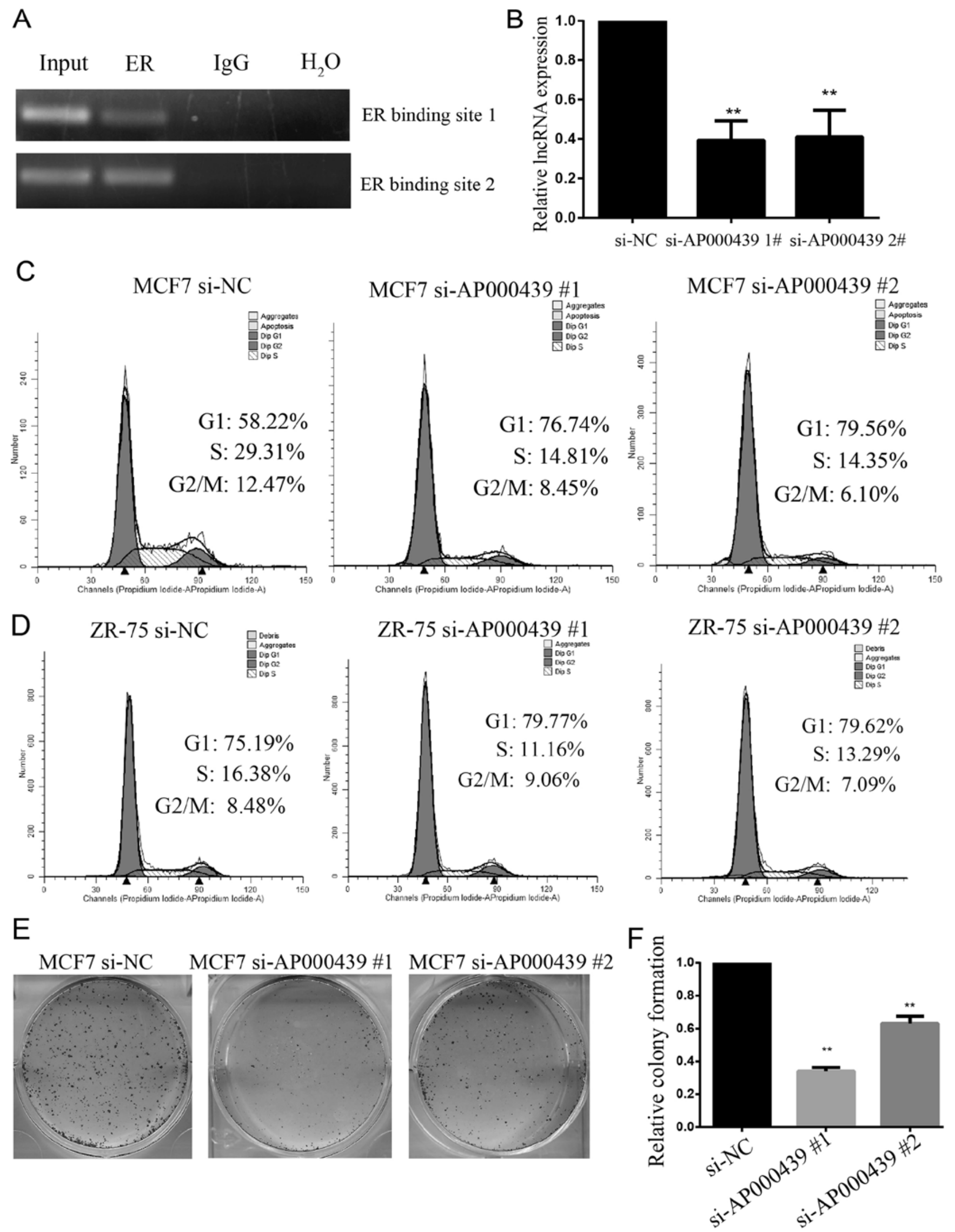

Figure 4. Silencing lncRNA AP000439.3 results in inhibition of cell cycle progression. (A) ChIP assays show that ER can bind upstream of AP000439.3. MCF7 chromatin fragments were immunoprecipitated with antibodies for ER and negative control antibody (normal rabbit IgG). (B) qPCR experiments show the knock down efficiency of individual siRNAs of AP000439.3. (C) Flow cytometry assay determinate the effect of silencing AP000439.3 on cell cycle progression in MCF7 cells. (D) Flow cytometry assay determined the effect of silencing AP000439.3 on cell cycle progression in in ZR-75-1 cells. (E) Representative results of colony formation assays of MCF7 cells transfected with indicated siRNAs. (F) Graphic display of results of colony formation experiments, the numbers of colonies were normalized to control sample, Means \pm SD of three independent experiments are shown. 


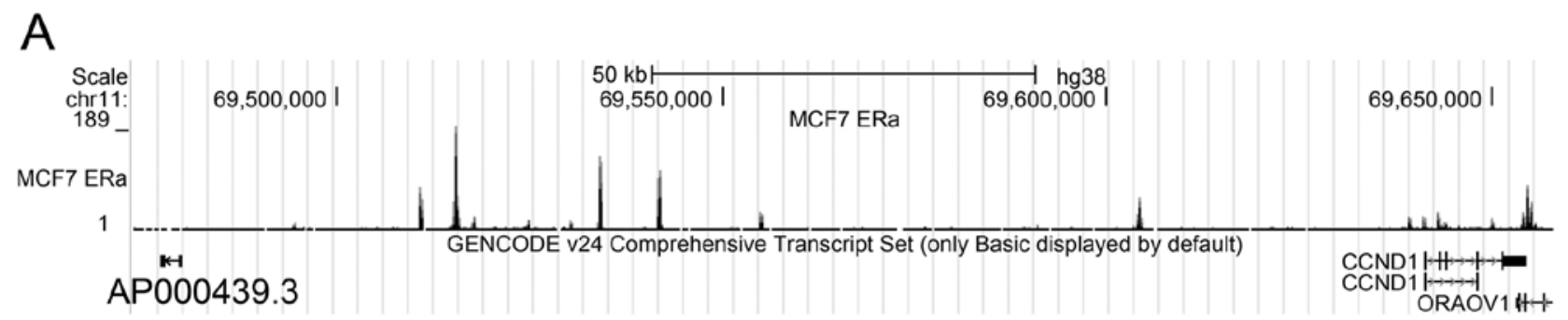

B

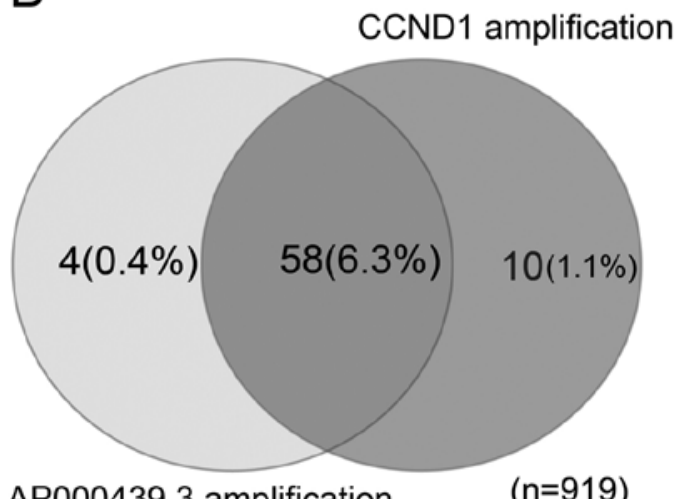

AP000439.3 amplification

$\mathrm{D}$

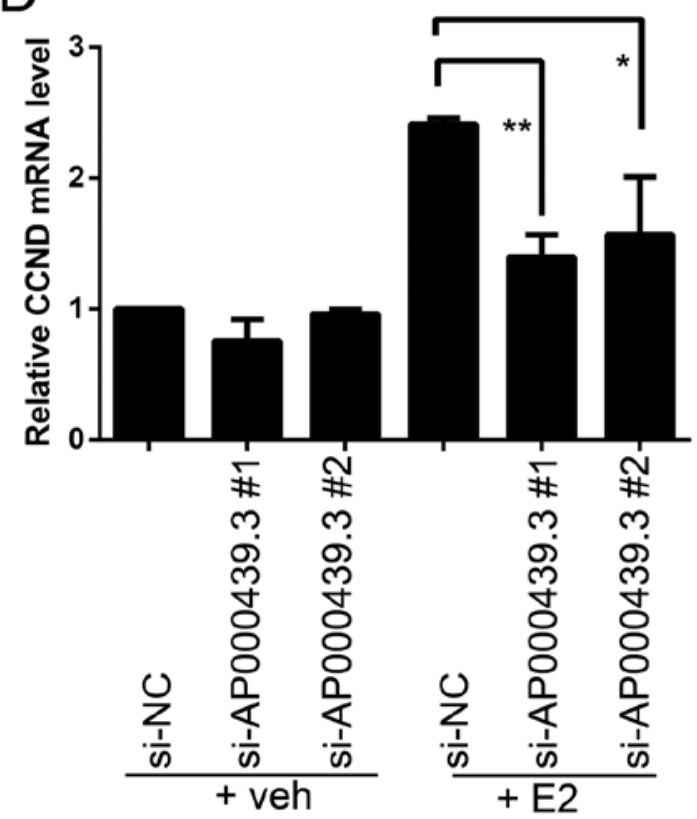

$\mathrm{C}$

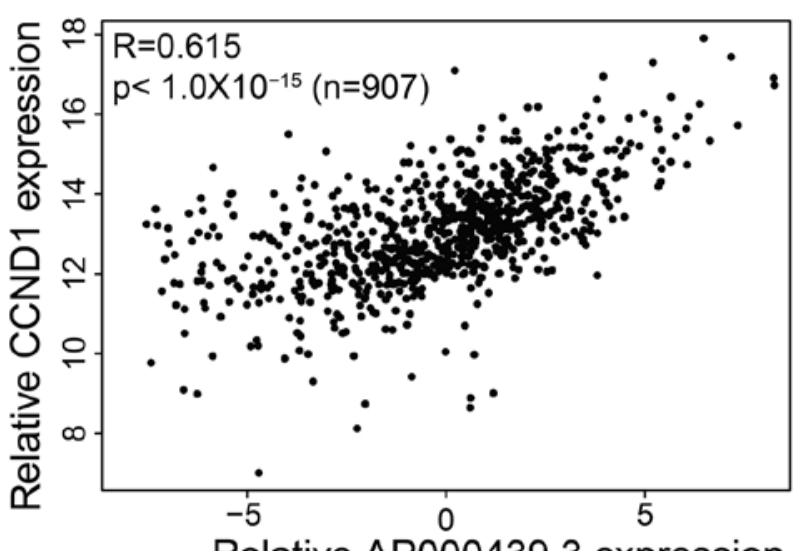

$\mathrm{E}$

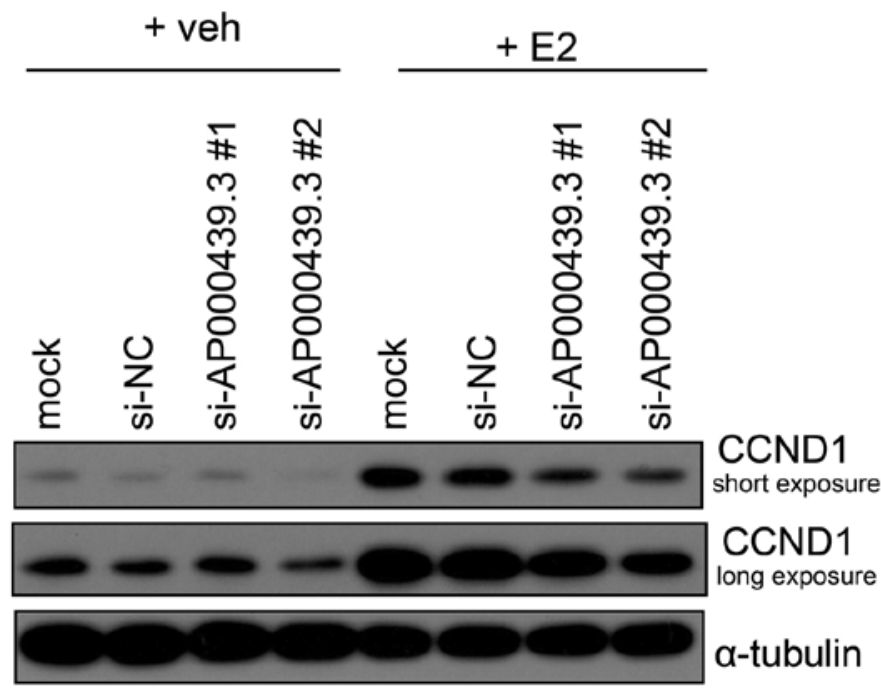

Figure 5. AP000439.3 facilitates estrogen induced CCDN1 expression. (A) Genomic organization of AP000439.3 and CCDN1. The direction of transcription are shown by the arrows, the ER $\alpha$ binding sites obtained by ChIP-seq are shown by the peaks. (B) Co-amplification of $A P 000439.3$ and $C C D N 1$. Left circle showing AP000439.3 amplified samples, right circle showing CCDN1 amplified and overlapped region showing co-amplified samples (COSMIC CNV data, $\mathrm{n}=919$ ). (C) Correlation between $C C D N 1$ and AP000439.3 expression (TCGA data). Pearson's product moment correlation coefficient was used to determinate association between $C C N D 1$ and $A P 000439.3$. (D) MCF7 were cultured in estrogen deprived medium and transfected with either AP000439.3 or control siRNAs for $48 \mathrm{~h}$, then treated with $10 \mathrm{nM}$ E2 for $8 \mathrm{~h}$. CCND1 mRNA level were detected by qPCR. (E) MCF7 were treated the same as (D), protein was analyzed by western blotting.

an ER-target lncRNA in a previous study $(13,14)$. Failure to observe a decrease of LINC01016 when silencing ER $\alpha$ may be due to its low expression level in T47D, hence hard to downregulate further, but easy to upregulate.

ER-regulated IncRNA AP000439.3 promotes cell cycle progression. To further characterize these ER-regulated
lncRNAs, we analyzed the expression of lncRNAs in breast tissues using The Cancer Genome Atlas (TCGA) data (31). Most of these selected lncRNAs were dramatically overexpressed in $\mathrm{ER}^{+}$breast cancer compared to $\mathrm{ER}^{-}$breast cancer and normal breast tissues ( $\mathrm{p}<0.001$, Fig. 3).

To explore the roles of ER target lncRNAs in breast cancer, we selected one of the most significantly upregulated lncRNA 


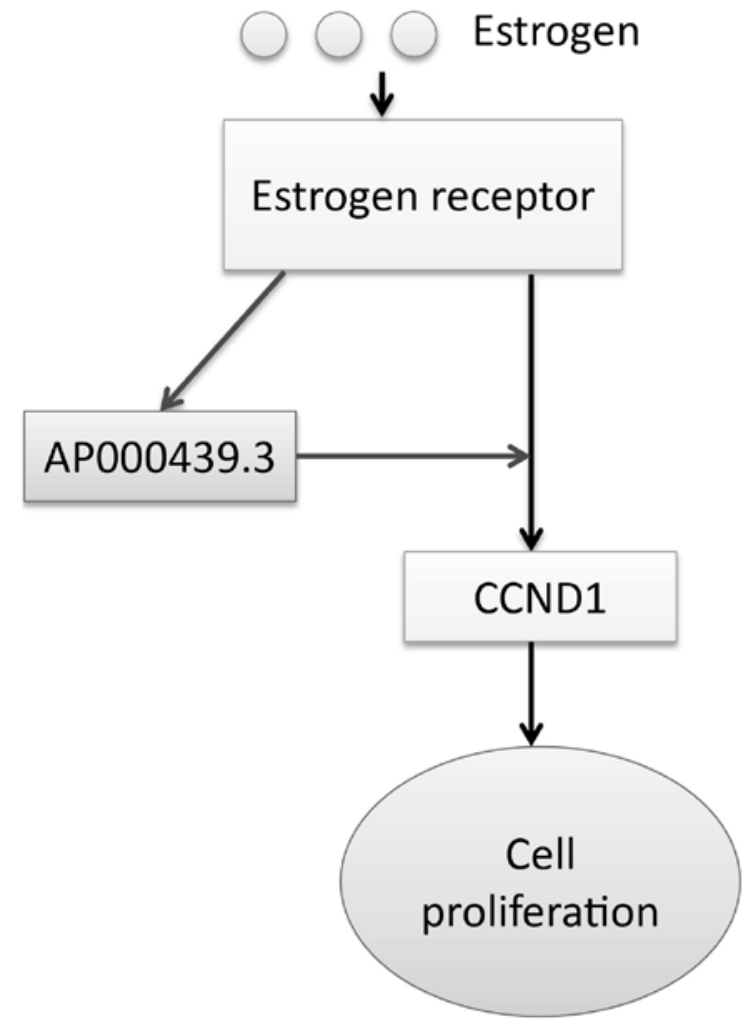

Figure 6. Model of regulation and roles of IncRNA AP000439.3. AP000439.3 is transcriptionally regulated by ER, AP000439.3 can improve the expression of $C C N D 1$ induced by estrogen and thus promote cell cycle progression and cell proliferation.

(AP000439.3) for further analysis. Using ChIPBase, an integrated database for decoding transcriptional regulators of genes and lncRNAs $(32,33)$, we found $E R \alpha$ is the top ranked regulator. To verify that AP000439.3 is regulated by ER, ChIP PCR assay was performed on potential ER binding sites identified by ChIP-seq data and ChIPBase. As shown in Fig. 4A, ER can directly bind upstream of AP000439.3.

Since ER can regulate dozens of genes that play crucial roles in breast cancer, we speculate these IncRNAs may also have an important impact on breast cancer cell function. AP000439.3 was silenced using two individual siRNAs by $>50 \%$ (Fig. 4B). The most dominant role of ER in breast cancer is promotion of cell proliferation and cell cycle progression (1). Hence the cell cycle progression was detected using flow cytometry assay. Silencing AP000439.3 resulted in a dramatic inhibition of the G1-S transition in MCF7 cells (Fig. 4C) and in ZR-75-1 cells (Fig. 4D). Likewise, silencing AP000439.3 significantly suppressed clonogenic proliferation (Fig. 4E and F). Taken together, IncRNA AP000439.3 is stimulated by activated ER, it is overexpressed in $\mathrm{ER}^{+}$breast cancer and promotes cell cycle progression and proliferation.

AP000439.3 facilitates estrogen induced CCDN1 expression. To understand how AP000439.3 regulates the cell cycle, we first investigated its location and gene structure. AP000439.3 is a long intergenic non-coding RNA (lincRNA) located at chromosome 11q13. AP000439.3 is $160 \mathrm{~kb}$ upstream of the $C C D N 1$ transcriptional start site (TSS) (Fig. 5A), AP000439.3 and CCDN1 are transcribed divergently (head to head). CCNDI encodes the cyclin D1 protein that serves as a regulator of cyclin-dependent kinase as a crucial regulator of the cell cycle. Amplification of $C C N D 1$ has been reported in many kinds of tumors including breast cancer, its amplification is associated with a poor prognosis (34). Analysis of COSMIC CNV data (35) revealed that CCND1 amplification occurred in 68/919 (7.4\%) breast cancer samples, 58 of $68(85 \%)$ of these also have an amplification of AP000439.3 (Fig. 5B). Expression analysis of $C C N D 1$ and $A P 000439.3$ showed a high corre-

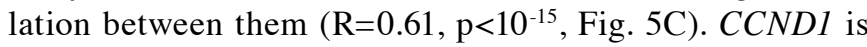
overexpressed in $\sim 50 \%$ breast cancer, while amplification of the $C C N D 1$ gene is present only in a minority of $C C N D 1$ overexpressed breast cancers (36), directly regulated by ER is another important reason for overexpression of CCND1 (37).

LncRNAs are likely to regulate the expression of an adjacent gene (38-40). Hence we speculated that AP000439.3 may influence the expression of CCND1. We silenced AP000439.3 in MCF7 and then treated these cells with either $\mathrm{E} 2$ or vehicle. Both qPCR and western blotting showed that knockdown of AP000439.3 could impair CCND1 expression induced by E2 (Fig. 5D). Taken together, these data suggest ER-regulated IncRNA AP000439.3 can facilitate ER regulation of $C C N D 1$ and thus promote cell cycle progression (Fig. 6).

\section{Discussion}

In this study, we described a genome-wide identification and characterization of ER-regulated lncRNAs in breast cancer cells. We found many of these lncRNAs were overexpressed in $\mathrm{ER}^{+}$breast cancer and co-expressed with some key regulators. Moreover, we found one of the most prominent lncRNA, AP000439.3, can promote cell cycle progression through enhancing $C C D N 1$ expression induced by estrogen.

The mechanisms of how AP000439.3 facilitate CCNDI expression remains to be further investigated. Considering AP000439.3 can influence transcription of CCND1, it may involve changes in chromatin organization. This mechanism have been reported by lncRNA CCAT1-L regulation on its adjacent gene C-MYC in colorectal cancer (41).

The roles of ER is mediated primarily by its downstream effectors, the ER-regulated protein-coding genes and their roles have been well studied. However, some important issues such as the mechanisms by which ER-mediated drug resistance remains largely unknown (42). Study of ER-regulated lncRNAs may provide a unique prospective to answer these questions.

Our findings may provide another layer of estrogenic control of gene expression: ER can promote expression of IncRNAs that are adjacent to protein-coding genes; these lncRNAs can serve as positive regulators that further facilitate transcriptional regulation by ER. It remains to be further investigated how many lncRNAs function in this way and whether these lncRNAs specifically regulate their neighboring adjacent genes or have a more extensive impact on ER regulation. Since these ER-regulated lncRNAs showed highly cell-type specificity, some oncogenic lncRNAs can be potential biomarkers and therapeutic targets. 


\section{Acknowledgements}

We would like to thank Ms. Qing Wei and Ms. Fang Su for flow cytometry analysis. We thank The Cancer Genome Atlas (TCGA) project and their contributors for providing this valuable public data set. This study was supported by grants from the Natural Science Foundation of China (81572484, 81420108026 and 81621004 to D.Y.); Guangdong Science and Technology Department (2014A050503026 to D.Y., 2015B050501004 and S2012030006287); Guangzhou Bureau of Science and Information Technology (201704030036 to D.Y. This study was also supported by the RNA Biology Center at the Cancer Science Institute of Singapore, NUS, as part of funding under the Singapore Ministry of Education's Tier 3 grants, grant no. MOE2014-T3-1-006.

\section{References}

1. Liang J and Shang Y: Estrogen and cancer. Annu Rev Physiol 75 225-240, 2013.

2. Marino M, Galluzzo P and Ascenzi P: Estrogen signaling multiple pathways to impact gene transcription. Curr Genomics 7: 497-508, 2006.

3. Yager JD and Davidson NE: Estrogen carcinogenesis in breast cancer. N Engl J Med 354: 270-282, 2006.

4. Shao W and Brown M: Advances in estrogen receptor biology: Prospects for improvements in targeted breast cancer therapy. Breast Cancer Res 6: 39-52, 2004.

5. Frasor J, Danes JM, Komm B, Chang KC, Lyttle CR and Katzenellenbogen BS: Profiling of estrogen up- and down-regulated gene expression in human breast cancer cells: Insights into gene networks and pathways underlying estrogenic control of proliferation and cell phenotype. Endocrinology 144: 4562-4574, 2003.

6. Coser KR, Chesnes J, Hur J, Ray S, Isselbacher KJ and Shioda T: Global analysis of ligand sensitivity of estrogen inducible and suppressible genes in MCF7/BUS breast cancer cells by DNA microarray. Proc Natl Acad Sci USA 100: 13994-13999, 2003.

7. Inoue A, Yoshida N, Omoto Y, Oguchi S, Yamori T, Kiyama R and Hayashi S: Development of cDNA microarray for expression profiling of estrogen-responsive genes. J Mol Endocrinol 29: $175-192,2002$

8. Soulez M and Parker MG: Identification of novel oestrogen receptor target genes in human ZR75-1 breast cancer cells by expression profiling. J Mol Endocrinol 27: 259-274, 2001.

9. Lin CY, Ström A, Vega VB, Kong SL, Yeo AL, Thomsen JS Chan WC, Doray B, Bangarusamy DK, Ramasamy A, et al: Discovery of estrogen receptor alpha target genes and response elements in breast tumor cells. Genome Biol 5: R66, 2004.

10. Tseng YY, Moriarity BS, Gong W, Akiyama R, Tiwari A, Kawakami H, Ronning P, Reuland B, Guenther K, Beadnell TC, et al: PVT1 dependence in cancer with MYC copy-number increase. Nature 512: 82-86, 2014.

11. Liu B, Sun L, Liu Q, Gong C, Yao Y, Lv X, Lin L, Yao H, Su F, Li D, et al: A cytoplasmic NF- $\kappa \mathrm{B}$ interacting long noncoding RNA blocks I $\kappa \mathrm{B}$ phosphorylation and suppresses breast cancer metastasis. Cancer Cell 27: 370-381, 2015.

12. Xue X, Yang YA, Zhang A, Fong KW, Kim J, Song B, Li S, Zhao JC and Yu J: LncRNA HOTAIR enhances ER signaling and confers tamoxifen resistance in breast cancer. Oncogene 35 2746-2755, 2016

13. Miano V, Ferrero G, Reineri S, Caizzi L, Annaratone L, Ricci L, Cutrupi S, Castellano I, Cordero F and De Bortoli M: Luminal long non-coding RNAs regulated by estrogen receptor alpha in a ligand-independent manner show functional roles in breast cancer. Oncotarget 7: 3201-3216, 2016.

14. Jonsson P, Coarfa C, Mesmar F, Raz T, Rajapakshe K, Thompson JF, Gunaratne PH and Williams C: Single-molecule sequencing reveals estrogen-regulated clinically relevant lncRNAs in breast cancer. Mol Endocrinol 29: 1634-1645, 2015.

15. Qiu JJ, Ye LC, Ding JX, Feng WW, Jin HY, Zhang Y, Li Q and Hua KQ: Expression and clinical significance of estrogenregulated long non-coding RNAs in estrogen receptor $\alpha$-positive ovarian cancer progression. Oncol Rep 31: 1613-1622, 2014.
16. Dago DN, Scafoglio C, Rinaldi A, Memoli D, Giurato G, Nassa G, Ravo M, Rizzo F, Tarallo R and Weisz A: Estrogen receptor beta impacts hormone-induced alternative mRNA splicing in breast cancer cells. BMC Genomics 16: 367, 2015.

17. Dobin A, Davis CA, Schlesinger F, Drenkow J, Zaleski C, Jha S, Batut P, Chaisson $M$ and Gingeras TR: STAR: Ultrafast universal RNA-seq aligner. Bioinformatics 29: 15-21, 2013.

18. Li B and Dewey CN: RSEM: Accurate transcript quantification from RNA-Seq data with or without a reference genome. BMC Bioinformatics 12: 323, 2011

19. Anders $\mathrm{S}$ and Huber W: Differential expression analysis for sequence count data. Genome Biol 11: R106, 2010.

20. Robinson MD, McCarthy DJ and Smyth GK: edgeR: A Bioconductor package for differential expression analysis of digital gene expression data. Bioinformatics 26: 139-140, 2010.

21. Franco HL, Nagari A and Kraus WL: TNFa signaling exposes latent estrogen receptor binding sites to alter the breast cancer cell transcriptome. Mol Cell 58: 21-34, 2015.

22. Langmead B, Trapnell C, Pop M and Salzberg SL: Ultrafast and memory-efficient alignment of short DNA sequences to the human genome. Genome Biol 10: R25, 2009.

23. Zhang Y, Liu T, Meyer CA, Eeckhoute J, Johnson DS, Bernstein BE, Nusbaum C, Myers RM, Brown M, Li W, et al: Model-based analysis of ChIP-Seq (MACS). Genome Biol 9: R137, 2008.

24. Li J, Han L, Roebuck P, Diao L, Liu L, Yuan Y, Weinstein JN and Liang H: TANRIC: An interactive open platform to explore the function of lncRNAs in cancer. Cancer Res 75: 3728-3737, 2015.

25. Dong S, Han J, Chen H, Liu T, Huen MS, Yang Y, Guo C and Huang J: The human SRCAP chromatin remodeling complex promotes DNA-end resection. Curr Biol 24: 2097-2110, 2014.

26. Derrien T, Johnson R, Bussotti G, Tanzer A, Djebali S, Tilgner H, Guernec G, Martin D, Merkel A, Knowles DG, et al: The GENCODE v7 catalog of human long noncoding RNAs: Analysis of their gene structure, evolution, and expression. Genome Res 22: 1775-1789, 2012.

27. Ulitsky I and Bartel DP: lincRNAs: Genomics, evolution, and mechanisms. Cell 154: 26-46, 2013.

28. Cabili MN, Trapnell C, Goff L, Koziol M, Tazon-Vega B, Regev A and Rinn JL: Integrative annotation of human large intergenic noncoding RNAs reveals global properties and specific subclasses. Genes Dev 25: 1915-1927, 2011.

29. Amin V, Harris RA, Onuchic V, Jackson AR, Charnecki T, Paithankar S, Lakshmi Subramanian S, Riehle K, Coarfa C and Milosavljevic A: Epigenomic footprints across 111 reference epigenomes reveal tissue-specific epigenetic regulation of lincRNAs. Nat Commun 6: 6370, 2015.

30. Li J, Lu Y, Akbani R, Ju Z, Roebuck PL, Liu W, Yang JY, Broom BM, Verhaak RG, Kane DW, et al: TCPA: A resource for cancer functional proteomics data. Nat Methods 10: 1046-1047, 2013.

31. Weinstein JN, Collisson EA, Mills GB, Shaw KR, Ozenberger BA, Ellrott K, Shmulevich I, Sander C and Stuart JM; Cancer Genome Atlas Research Network: The Cancer Genome Atlas Pan-Cancer analysis project. Nat Genet 45: 1113-1120, 2013.

32. Yang JH, Li JH, Jiang S, Zhou H and Qu LH: ChIPBase: A database for decoding the transcriptional regulation of long noncoding RNA and microRNA genes from ChIP-Seq data. Nucleic Acids Res 41D: D177-D187, 2013.

33. Zhou KR, Liu S, Sun WJ, Zheng LL, Zhou H, Yang JH and Qu LH: ChIPBase v2.0: Decoding transcriptional regulatory networks of non-coding RNAs and protein-coding genes from ChIP-seq data. Nucleic Acids Res 45D: D43-D50, 2017.

34. Musgrove EA, Caldon CE, Barraclough J, Stone A and Sutherland RL: Cyclin D as a therapeutic target in cancer. Nat Rev Cancer 11: 558-572, 2011.

35. Forbes SA, Bindal N, Bamford S, Cole C, Kok CY, Beare D, Jia M, Shepherd R, Leung K, Menzies A, et al: COSMIC: Mining complete cancer genomes in the Catalogue of Somatic Mutations in Cancer. Nucleic Acids Res 39 (Database): D945-D950, 2011.

36. Arnold A and Papanikolaou A: Cyclin D1 in breast cancer pathogenesis. J Clin Oncol 23: 4215-4224, 2005.

37. Eeckhoute J, Carroll JS, Geistlinger TR, Torres-Arzayus MI and Brown M: A cell-type-specific transcriptional network required for estrogen regulation of cyclin D1 and cell cycle progression in breast cancer. Genes Dev 20: 2513-2526, 2006.

38. Berghoff EG, Clark MF, Chen S, Cajigas I, Leib DE and Kohtz JD: Evf2 (Dlx6as) lncRNA regulates ultraconserved enhancer methylation and the differential transcriptional control of adjacent genes. Development 140: 4407-4416, 2013. 
39. Spurlock CF III, Tossberg JT, Guo Y, Collier SP, Crooke PS III and Aune TM: Expression and functions of long noncoding RNAs during human Thelper cell differentiation. Nat Commun 6: 6932, 2015.

40. Luo S, Lu JY, Liu L, Yin Y, Chen C, Han X, Wu B, Xu R, Liu W, Yan $\mathrm{P}$, et al: Divergent lncRNAs regulate gene expression and lineage differentiation in pluripotent cells. Cell Stem Cell 18: 637-652, 2016
41. Xiang JF, Yin QF, Chen T, Zhang Y, Zhang XO, Wu Z, Zhang S, Wang HB, Ge J, Lu X, et al: Human colorectal cancer-specific CCAT1-L lncRNA regulates long-range chromatin interactions at the MYC locus. Cell Res 24: 513-531, 2014.

42. Xu CY, Jiang ZN, Zhou Y, Li JJ and Huang LM: Estrogen receptor $\alpha$ roles in breast cancer chemoresistance. Asian Pac J Cancer Prev 14: 4049-4052, 2013. 